\title{
Effect of Population Density and Nutrition on Nitrate Reductase Activity in Seed Guar Varieties
}

\author{
M. Tagore Naik, D. Srihari and A.V.D. Dorajeerao*
}

Horticultural Research Station, Mahanandi College of Horticulture,

Venkataramannagudem, Andhra Pradesh, India

*Corresponding author

\section{A B S T R A C T}

\begin{tabular}{|c|c|}
\hline Keywords & \\
\hline $\begin{array}{l}\text { Guar, Population } \\
\text { density, Nutrition. }\end{array}$ & \multirow{3}{*}{$\begin{array}{l}\text { The effect of planting geometry and nutrition on growth and flowering of } \\
\text { seed guar cultivars viz., } \mathrm{HG} 365 \text { and } \mathrm{HG} 563 \text { was analysed under } \\
\text { Mahanandi conditions. The nitrate reductase activity, crude gum }(\%) \text {, crude } \\
\text { protein }(\%) \text {, seed yield per plant }(\mathrm{g}) \text { was highest in variety } \mathrm{HG} 365 \text {, } \\
\text { planting geometry of } 30 \mathrm{~cm} \times 20 \mathrm{~cm}\left(\mathrm{~S}_{2}\right) \text { and Nutrition dose of } 45 \mathrm{~N}: 60 \mathrm{P} \text { : } \\
60 \mathrm{~K} \text { : } 30 \mathrm{~S} \mathrm{~kg} \text { per ha }\left(\mathrm{F}_{3}\right) \text {. }\end{array}$} \\
\hline Article Info & \\
\hline $\begin{array}{l}\text { Accepted: } \\
28 \text { October } 2017 \\
\text { Available Online: } \\
\text { 10 December } 2017\end{array}$ & \\
\hline
\end{tabular}

\section{Introduction}

Cluster bean is botanically called as Cyamopsis tetragonoloba (L.) Taub. It belongs to the family Leguminaceae. The crop is popularly known as guar referring to its seed. India is considered as native place for guar or cluster bean. It has been used as vegetable in our country from hundreds of years. The crop is renowned as drought hardy, being deep rooted and having a low water requirement. It requires a low annual rainfall of about $400 \mathrm{~mm}$ to $500 \mathrm{~mm}$. Guar tolerates high temperature and dry conditions, thus gaining popularity in arid and semi-arid climates (Undersander et al., 2006). The present experiment has been conducted to elucidate the effect of population density and nutrition on nitrate reductase activity in seed guar varieties

\section{Materials and Methods}

The experiment was conducted in factorial randomized design with three factors viz., varieties (2), planting geometry levels (3) and nutritional levels (3) replicated thrice. The plot was laid out at Horticultural Research Station, Mahanandi, Kurnool district of Andhra Pradesh during both kharif and rabi seasons of the years 2014-15 and 2015-16. The data obtained from both the years was pooled and presented in the tables.

\section{Results and Discussion}

Nitrate reductase activity ( $\mu$ moles $\mathrm{NO}_{2} \mathrm{~g}$ fresh weight ${ }^{-1} \mathbf{h r}^{-1}$ )

The data on nitrate reductase activity (Table 1) revealed that there were significant 
differences due to variety, planting geometry, nutritional combinations and their interactions. Among the varieties HG 365 recorded the highest nitrate reductase activity both in kharif (80.64) and rabi seasons (71.77). Planting geometry of $30 \mathrm{~cm} \mathrm{x} 20 \mathrm{~cm}$ $\left(\mathrm{S}_{2}\right)$ recorded significantly highest nitrate reductase activity (kharif 76.25 ; rabi67.86) on followed by $40 \mathrm{~cm} \times 10 \mathrm{~cm}\left(\mathrm{~S}_{3}\right)$ (kharif 73.45; rabi65.37). Application of $45 \mathrm{~N}: 60 \mathrm{P}: 60 \mathrm{~K}$ : $30 \mathrm{~S} \mathrm{~kg}$ per ha $\left(\mathrm{F}_{3}\right)$ recorded the highest nitrate reductase activity (kharif 78.38; rabi 69.76) which was on par with $30 \mathrm{~N}: 40 \mathrm{P}: 40 \mathrm{~K}: 20 \mathrm{~S}$ kg per ha $\left(\mathrm{F}_{2}\right)$ (kharif 76.05 ; rabi 67.68).

\section{Crude protein $(\%)$}

Significant differences were observed in the crude protein (Table 2) due to variety, planting geometry, nutritional combinations and their interactions. Among the varieties HG 365 recorded the highest crude protein both in kharif $(24.34 \%)$ and rabi seasons $(23.13 \%)$. Planting geometry of $30 \mathrm{~cm} \times 20$ $\mathrm{cm}\left(\mathrm{S}_{2}\right)$ recorded significantly the highest crude protein (kharif: $24.71 \%$; rabi: $23.48 \%$ ) followed by $40 \mathrm{~cm} \times 10 \mathrm{~cm}\left(\mathrm{~S}_{3}\right)$ (kharif: 23.10 \%; rabi: $21.95 \%$ ). Application of 45N: 60P: 60K: $30 \mathrm{~S}$ kg per ha $\left(\mathrm{F}_{3}\right)$ recorded the highest crude protein (kharif24.00\%; rabi22.80\%) which was on par with 30N: 40P: 40K: $20 \mathrm{~S}$ kg per ha $\left(\mathrm{F}_{2}\right)$ (kharif23.51\%; rabi22.33\%). The interaction effect of planting geometry plus nutrient level was found significant both during kharif and rabi. The highest crude protein was recorded by the planting pattern of $30 \mathrm{~cm} \times 20 \mathrm{~cm}$ plus application of $45 \mathrm{~N}$ : 60P: 60K: $30 \mathrm{~S} \mathrm{~kg}$ per ha (kharif25.31; rabi24.04) and HG $365+$ application of $45 \mathrm{~N}$ : 60P: 60K: $30 \mathrm{~S} \mathrm{~kg}$ per ha (kharif26.01; rabi24.71).

It is observed from the results on nitrate reducase activity and crude protein that both of them were associated together. Higher the nitrate reductase activity higher was the crude protein content and vice versa. Thus in the present study both these values were recorded at maximum level by the variety HG 365 in combination with planting geometry at $30 \mathrm{~cm}$ x $20 \mathrm{~cm}$ (the lowest population density) and the highest level nutritional combination at 45N: 60P: 60K: 30S. The enzyme nitrate reductase helps in the assimilation of nitrate nitrogen into the plants and therefore it might help in higher nitrogen content in various plant parts making it available for the biosynthesis of protein molecules. This might be the reason as to why a higher activity of nitrate reductase as observed in the variety HG 365 in combination with planting geometry at $30 \mathrm{~cm} \times 20 \mathrm{~cm}$ (the lowest population density) and the highest level nutritional combination at $45 \mathrm{~N}$ : $60 \mathrm{P}: 60 \mathrm{~K}$ : $30 \mathrm{~S}$ might have led to a higher content of crude protein. However, it was observed that both the activity of enzyme and content of protein did not increase significantly with increase in nutrient application from the level of $30 \mathrm{~N}: 40 \mathrm{P}: 40 \mathrm{~K}: 20 \mathrm{~S}$ to the level of $45 \mathrm{~N}$ : 60P: 60K: 30S thus making the effect of additional amount of nutrients null and void. However, the additional dose of nutrients were found beneficial provided there was an increase in the population density as evident from the comparison between these values recorded from the highest population density coupled with highest dose and lowest population densities supplied with highest dose.

The increase in protein content at high nutritional dose could be due to a high $\mathrm{S}$ content, since the nutrient sulphur is an important constituent of some amino acid molecules and therefore resulted in increases in protein content at higher doses (Baviskar et al., 2012). Singh et al., (2006) was also of the same opinion.

According to Yadav (2011) the synergistic effect of $\mathrm{P}$ and $\mathrm{S}$ may be due to utilization of 
high quantities of nutrients through their welldeveloped root system and nodules which might have resulted in better growth and yield. Increasing doses of sulphur application resulted in a significant increase in protein content of cluster bean. The positive response to added sulphur is assigned to low status of available $S$ in the soil or due to stimulating effect of applied sulphur in the synthesis of chloroplast protein resulting in greater photosynthetic efficiency which in turn got translated in term of increased yield. In his studies, nitrogen content was significantly increased with the increase in level of $\mathrm{P}$ and S. Dwivedi and Bapat (1998) reported that nitrogen content in soybean increased significantly by $\mathrm{P}$ and $\mathrm{S}$ application up to 50 $\mathrm{kg} \mathrm{ha}^{-1}$ of each. The interaction of $\mathrm{P}$ and $\mathrm{S}$ was significant and maximum nitrogen content was recorded at the highest dose of $\mathrm{P}$ and $\mathrm{S}$. Protein content in cluster bean grain was increased significantly with application of $\mathrm{P}$ and $\mathrm{S}$ individually as well as in combination. The maximum increase in protein content obtained with the highest $\mathrm{P}$ and $\mathrm{S}$ was attributed to more nitrogen fixation. Similar results were also reported by Shankaralingappa et al., (2000) in cowpea and Kumawat et al., (2004) in taramira. Increasing doses of sulphur application resulted in a significant increase in protein content of cluster bean. The positive response to added sulphur is assigned to low status of available $S$ in soil or due to stimulating effect of applied sulphur in the synthesis of chloroplast protein resulting in greater photosynthetic efficiency which in turn translated in term of increased yield.

The maximum crude protein content was obtained when nitrogen was applied at higher dose. The higher crude protein at higher nitrogen levels was felt mainly due to structural role of nitrogen in building up amino acids. The progressive increase in crude protein contents with increasing nitrogen rates were also reported by Kumawat et al., (2000), Sheikh(2004), Morshed et al., (2008) and Ibrahim (2009).

\section{Crude gum (\%)}

Significant differences were observed in the crude gum (Table 3) due to variety, planting geometry, nutritional combinations and some of their interactions. Among the varieties HG 365 recorded the highest crude gum both in kharif $(32.76 \%)$ and rabi seasons (32.40\%).

Planting geometry of $30 \mathrm{~cm} \times 20 \mathrm{~cm}\left(\mathrm{~S}_{2}\right)$ recorded significantly the highest crude gum (kharif $33.72 \%$; rabi $33.35 \%$ ) followed by $40 \mathrm{~cm} \times 10 \mathrm{~cm}\left(\mathrm{~S}_{3}\right)$ (kharif $31.43 \%$; rabi $31.08 \%$ ). Application of $45 \mathrm{~N}: 60 \mathrm{P}: 60 \mathrm{~K}: 30 \mathrm{~S}$ $\mathrm{kg}$ per ha $\left(\mathrm{F}_{3}\right)$ recorded the highest crude gum (kharif $33.36 \%$; rabi $32.99 \%$ ) which was on par with $30 \mathrm{~N}$ : 40P: $40 \mathrm{~K}: 20 \mathrm{~S} \mathrm{~kg}$ per ha $\left(\mathrm{F}_{2}\right)$ (kharif $31.59 \%$; rabi32.14\%). The content of crude gum in the seed governs the value of the guar produce since the gum is the product of export value.

Among the varieties HG 365 exhibited the highest gum content and among the planting geometry levels, the lowest population density at $30 \mathrm{~cm} \times 20 \mathrm{~cm}$ exhibited the highest gum content being the one having the bold sized seeds from significantly hefty pods and clusters. The individual plant being capable of drawing more nutrients and sufficient light over an extended period of duration spent in building up vegetative frame work as well as prolonged reproductive period, exhibited better quality parameters and therefore was also able to produce seeds with a higher content of galactomannan. The nutrient application at the highest level of 45N: 60P: 60K: $30 \mathrm{~S}$ was on par with $30 \mathrm{~N}$ : 40P: 40K: 20S thus making the effect of additional amount of nutrients non-significant in increasing gum content of individual seeds. 
Table.1 Nitrate reductase activity ( $\mu$ moles $\mathrm{NO}_{2} \mathrm{~g}_{\text {fresh }}$ weight ${ }^{-1} \mathrm{hr}^{-1}$ ) at $60 \mathrm{DAS}$ as influenced by variety, planting geometry and nutritional combination during kharif and rabi (pooled data of 2014-15 and 2015-16)

\begin{tabular}{|c|c|c|c|c|c|c|c|}
\hline \multirow{3}{*}{$\begin{array}{l}\text { Planting Geometry } \\
\text { (B) }\end{array}$} & \multirow{3}{*}{$\begin{array}{c}\text { Nutritional } \\
\text { Combination }(\mathrm{C})\end{array}$} & \multicolumn{6}{|c|}{ Variety $(\mathrm{A})$} \\
\hline & & \multicolumn{3}{|c|}{ Kharif } & \multicolumn{3}{|c|}{ Rabi } \\
\hline & & HG 365 & HG 563 & Mean & $\begin{array}{l}\mathrm{HG} \\
365\end{array}$ & $\begin{array}{l}\mathrm{HG} \\
563\end{array}$ & Mean \\
\hline \multirow{4}{*}{$\begin{array}{l}\mathrm{S}_{1}(30 \mathrm{~cm} \times 10 \mathrm{~cm}) \\
\left(33.3 \text { plants per } \mathrm{m}^{2}\right)\end{array}$} & $\mathrm{F}_{1}(15 \mathrm{~N}: 20 \mathrm{P}: 20 \mathrm{~K}: 10 \mathrm{~S})$ & 78.51 & 59.16 & 68.84 & 69.88 & 52.65 & 61.26 \\
\hline & $\mathrm{F}_{2}(30 \mathrm{~N}: 40 \mathrm{P}: 40 \mathrm{~K}: 20 \mathrm{~S})$ & 80.16 & 68.14 & 74.15 & 71.34 & 60.64 & 65.99 \\
\hline & $\mathrm{F}_{3}(45 \mathrm{~N}: 60 \mathrm{P}: 60 \mathrm{~K}: 30 \mathrm{~S})$ & 81.53 & 69.30 & 75.42 & 72.57 & 61.68 & 67.12 \\
\hline & Mean & 80.07 & 65.53 & 72.80 & 71.26 & 58.32 & 64.79 \\
\hline \multirow{4}{*}{$\begin{array}{l}\mathrm{S}_{2}(30 \mathrm{~cm} \times 20 \mathrm{~cm}) \\
\left(16.7 \text { plants per } \mathrm{m}^{2}\right)\end{array}$} & $\mathrm{F}_{1}(15 \mathrm{~N}: 20 \mathrm{P}: 20 \mathrm{~K}: 10 \mathrm{~S})$ & 74.09 & 62.97 & 68.53 & 65.94 & 56.05 & 60.99 \\
\hline & $\mathrm{F}_{2}(30 \mathrm{~N}: 40 \mathrm{P}: 40 \mathrm{~K}: 20 \mathrm{~S})$ & 83.88 & 71.30 & 77.59 & 74.66 & 63.46 & 69.06 \\
\hline & $\mathrm{F}_{3}(45 \mathrm{~N}: 60 \mathrm{P}: 60 \mathrm{~K}: 30 \mathrm{~S})$ & 89.32 & 75.92 & 82.62 & 79.49 & 67.57 & 73.53 \\
\hline & Mean & 82.43 & 70.07 & 76.25 & 73.36 & 62.36 & 67.86 \\
\hline \multirow{4}{*}{$\begin{array}{c}\mathrm{S}_{3}(40 \mathrm{~cm} \times 10 \mathrm{~cm}) \\
\left(25 \text { plants per } \mathrm{m}^{2}\right)\end{array}$} & $\mathrm{F}_{1}(15 \mathrm{~N}: 20 \mathrm{P}: 20 \mathrm{~K}: 10 \mathrm{~S})$ & 72.27 & 61.43 & 66.85 & 64.32 & 54.67 & 59.49 \\
\hline & $\mathrm{F}_{2}(30 \mathrm{~N}: 40 \mathrm{P}: 40 \mathrm{~K}: 20 \mathrm{~S})$ & 82.60 & 70.21 & 76.40 & 73.51 & 62.49 & 68.00 \\
\hline & $\mathrm{F}_{3}(45 \mathrm{~N}: 60 \mathrm{P}: 60 \mathrm{~K}: 30 \mathrm{~S})$ & 83.35 & 70.85 & 77.10 & 74.18 & 63.06 & 68.62 \\
\hline & Mean & 79.41 & 67.49 & 73.45 & 70.67 & 60.07 & 65.37 \\
\hline \multicolumn{8}{|c|}{ For Comparing varieties (A) and Nutritional combinations (C) } \\
\hline \multicolumn{2}{|c|}{$\mathrm{F}_{1}(15 \mathrm{~N}: 20 \mathrm{P}: 20 \mathrm{~K}: 10 \mathrm{~S})$} & 74.96 & 61.18 & 68.07 & 66.71 & 54.45 & 60.58 \\
\hline \multicolumn{2}{|c|}{$\mathrm{F}_{2}(30 \mathrm{~N}: 40 \mathrm{P}: 40 \mathrm{~K}: 20 \mathrm{~S})$} & 82.21 & 69.88 & 76.05 & 73.17 & 62.20 & 67.68 \\
\hline \multicolumn{2}{|c|}{$\mathrm{F}_{3}(45 \mathrm{~N}: 60 \mathrm{P}: 60 \mathrm{~K}: 30 \mathrm{~S})$} & 84.74 & 72.03 & 78.38 & 75.41 & 64.10 & 69.76 \\
\hline \multicolumn{2}{|c|}{ Mean } & 80.64 & 67.70 & 74.17 & 71.77 & 60.25 & 66.01 \\
\hline Factor & \multicolumn{2}{|l|}{$S E m \pm$} & \multicolumn{2}{|l|}{$C D$} & \multicolumn{2}{|c|}{$S E m \pm$} & $C D$ \\
\hline $\operatorname{Variety}(A)$ & \multicolumn{2}{|l|}{1.83} & & 5.30 & \multicolumn{2}{|c|}{1.63} & 4.72 \\
\hline Ptg. Geom. (B) & \multicolumn{2}{|l|}{0.37} & & 1.06 & \multicolumn{2}{|c|}{0.33} & 0.94 \\
\hline Nutril. Combn.(C) & \multicolumn{2}{|l|}{1.08} & & 3.13 & \multicolumn{2}{|c|}{0.96} & 2.79 \\
\hline$A x B$ & \multicolumn{2}{|l|}{-} & & $N S$ & \multicolumn{2}{|c|}{-} & $N S$ \\
\hline$B x C$ & \multicolumn{2}{|l|}{1.38} & & 3.98 & \multicolumn{2}{|c|}{1.22} & 3.55 \\
\hline$A x C$ & \multicolumn{2}{|l|}{-} & & $N S$ & \multirow{2}{*}{\multicolumn{2}{|c|}{ - }} & $N S$ \\
\hline$A x B \times C$ & \multicolumn{2}{|l|}{ - } & & $N S$ & & & $N S$ \\
\hline
\end{tabular}

CD: CD at 5\% level of significance 
Table. 2 Crude protein (\%) as influenced by variety, planting geometry and nutritional combination during kharif and rabi (pooled data of 2014-15 and 2015-16)

\begin{tabular}{|c|c|c|c|c|c|c|c|}
\hline \multirow{3}{*}{$\begin{array}{l}\text { Planting Geometry } \\
\text { (B) }\end{array}$} & \multirow{3}{*}{$\begin{array}{l}\text { Nutritional Combination } \\
\text { (C) }\end{array}$} & \multicolumn{6}{|c|}{ Variety $(\mathrm{A})$} \\
\hline & & \multicolumn{3}{|c|}{ Kharif } & \multicolumn{3}{|c|}{ Rabi } \\
\hline & & HG 365 & HG 563 & Mean & HG 365 & HG 563 & Mean \\
\hline \multirow{4}{*}{$\begin{array}{l}S_{1}\left(30 \mathrm{~cm} \times 10 \mathrm{~cm}^{2}\right. \\
\left(33.3 \text { plants per } \mathrm{m}^{2}\right)\end{array}$} & $\mathrm{F}_{1}(15 \mathrm{~N}: 20 \mathrm{P}: 20 \mathrm{~K}: 10 \mathrm{~S})$ & 21.44 & 19.30 & 20.37 & 20.37 & 18.33 & 19.35 \\
\hline & $\mathrm{F}_{2}(30 \mathrm{~N}: 40 \mathrm{P}: 40 \mathrm{~K}: 20 \mathrm{~S})$ & 23.06 & 20.75 & 21.91 & 21.91 & 19.72 & 20.81 \\
\hline & $\mathrm{F}_{3}(45 \mathrm{~N}: 60 \mathrm{P}: 60 \mathrm{~K}: 30 \mathrm{~S})$ & 23.60 & 21.24 & 22.42 & 22.42 & 20.17 & 21.29 \\
\hline & Mean & 22.70 & 20.43 & 21.56 & 21.56 & 19.41 & 20.48 \\
\hline \multirow{4}{*}{$\begin{array}{l}\mathrm{S}_{2}(30 \mathrm{~cm} \times 20 \mathrm{~cm}) \\
\left(16.7 \text { plants per } \mathrm{m}^{2}\right)\end{array}$} & $\mathrm{F}_{1}(15 \mathrm{~N}: 20 \mathrm{P}: 20 \mathrm{~K}: 10 \mathrm{~S})$ & 25.10 & 22.59 & 23.84 & 23.84 & 21.46 & 22.65 \\
\hline & $\mathrm{F}_{2}(30 \mathrm{~N}: 40 \mathrm{P}: 40 \mathrm{~K}: 20 \mathrm{~S})$ & 26.31 & 23.67 & 24.99 & 24.99 & 22.49 & 23.74 \\
\hline & $\mathrm{F}_{3}(45 \mathrm{~N}: 60 \mathrm{P}: 60 \mathrm{~K}: 30 \mathrm{~S})$ & 26.64 & 23.97 & 25.31 & 25.31 & 22.77 & 24.04 \\
\hline & Mean & 26.01 & 23.41 & 24.71 & 24.71 & 22.24 & 23.48 \\
\hline \multirow{4}{*}{$\begin{array}{l}\mathrm{S}_{3}(40 \mathrm{~cm} \times 10 \mathrm{~cm}) \\
\left(25 \text { plants per } \mathrm{m}^{2}\right)\end{array}$} & $\mathrm{F}_{1}(15 \mathrm{~N}: 20 \mathrm{P}: 20 \mathrm{~K}: 10 \mathrm{~S})$ & 22.54 & 20.29 & 21.41 & 21.41 & 19.27 & 20.34 \\
\hline & $\mathrm{F}_{2}(30 \mathrm{~N}: 40 \mathrm{P}: 40 \mathrm{~K}: 20 \mathrm{~S})$ & 24.86 & 22.38 & 23.62 & 23.62 & 21.26 & 22.44 \\
\hline & $\mathrm{F}_{3}(45 \mathrm{~N}: 60 \mathrm{P}: 60 \mathrm{~K}: 30 \mathrm{~S})$ & 25.55 & 22.99 & 24.27 & 24.27 & 21.84 & 23.05 \\
\hline & Mean & 24.32 & 21.88 & 23.10 & 23.10 & 20.79 & 21.95 \\
\hline \multicolumn{8}{|c|}{ For Comparing varieties (A) and Nutritional combinations (C) } \\
\hline \multicolumn{2}{|c|}{$\mathrm{F}_{1}(15 \mathrm{~N}: 20 \mathrm{P}: 20 \mathrm{~K}: 10 \mathrm{~S})$} & 23.03 & 20.72 & 21.87 & 21.87 & 19.69 & 20.78 \\
\hline \multicolumn{2}{|c|}{$\mathrm{F}_{2}(30 \mathrm{~N}: 40 \mathrm{P}: 40 \mathrm{~K}: 20 \mathrm{~S})$} & 24.74 & 22.27 & 23.51 & 23.51 & 21.15 & 22.33 \\
\hline \multicolumn{2}{|c|}{$\mathrm{F}_{3}(45 \mathrm{~N}: 60 \mathrm{P}: 60 \mathrm{~K}: 30 \mathrm{~S})$} & 25.26 & 22.73 & 24.00 & 24.00 & 21.60 & 22.80 \\
\hline \multicolumn{2}{|c|}{ Mean } & 24.34 & 21.91 & 23.13 & 23.13 & 20.81 & 21.97 \\
\hline Factor & \multicolumn{2}{|l|}{ SEm士 } & \multicolumn{2}{|c|}{$C D$} & \multicolumn{2}{|c|}{ SEm+ } & $C D$ \\
\hline Variety (A) & & \multicolumn{2}{|c|}{1.00} & \multicolumn{2}{|c|}{0.33} & 0.95 \\
\hline Ptg. Geom. (B) & \multicolumn{2}{|l|}{0.31} & \multicolumn{2}{|c|}{0.91} & \multicolumn{2}{|c|}{0.30} & 0.87 \\
\hline Nutril. Combn.(C) & \multicolumn{2}{|l|}{0.22} & \multicolumn{2}{|c|}{$\begin{array}{c}0.64 \\
-15\end{array}$} & \multicolumn{2}{|c|}{0.21} & 0.61 \\
\hline$A \times B$ & \multicolumn{2}{|l|}{ - } & \multicolumn{2}{|c|}{$N S$} & \multicolumn{2}{|c|}{ - } & $N S$ \\
\hline $\mathbf{B} \times \mathbf{C}$ & \multicolumn{2}{|l|}{0.51} & \multicolumn{2}{|c|}{1.48} & \multicolumn{2}{|c|}{0.48} & 1.40 \\
\hline $\mathbf{A} \times \mathbf{C}$ & 0.54 & & 1.5 & & & & 1.48 \\
\hline$A \times B \times C$ & 0.84 & & 2.4 & & & & $N S$ \\
\hline
\end{tabular}

CD: $\mathrm{CD}$ at $5 \%$ level of significance 
Table.3 Crude gum (\%) as influenced by variety, planting geometry and nutritional combination during kharif and rabi (pooled data of 2014-15 and 2015-16)

\begin{tabular}{|c|c|c|c|c|c|c|c|}
\hline \multirow{3}{*}{$\begin{array}{l}\text { Planting Geometry } \\
\text { (B) }\end{array}$} & \multirow{3}{*}{$\begin{array}{c}\text { Nutritional Combination } \\
\text { (C) }\end{array}$} & \multicolumn{6}{|c|}{ Variety $(\mathrm{A})$} \\
\hline & & \multicolumn{3}{|c|}{ Kharif } & \multicolumn{3}{|c|}{ Rabi } \\
\hline & & HG 365 & HG 563 & Mean & HG 365 & HG 563 & Mean \\
\hline \multirow{4}{*}{$\begin{array}{l}\mathrm{S}_{1}(30 \mathrm{~cm} \times 10 \mathrm{~cm}) \\
\left(33.3 \text { plants per } \mathrm{m}^{2}\right)\end{array}$} & $\mathrm{F}_{1}(15 \mathrm{~N}: 20 \mathrm{P}: 20 \mathrm{~K}: 10 \mathrm{~S})$ & 29.22 & 27.61 & 28.41 & 28.90 & 27.31 & 28.10 \\
\hline & $\mathrm{F}_{2}(30 \mathrm{~N}: 40 \mathrm{P}: 40 \mathrm{~K}: 20 \mathrm{~S})$ & 32.02 & 30.27 & 31.14 & 31.67 & 29.93 & 30.80 \\
\hline & $\mathrm{F}_{3}(45 \mathrm{~N}: 60 \mathrm{P}: 60 \mathrm{~K}: 30 \mathrm{~S})$ & 32.67 & 30.89 & 31.78 & 32.31 & 30.55 & 31.43 \\
\hline & Mean & 31.30 & 29.59 & 30.45 & 30.96 & 29.26 & 30.11 \\
\hline \multirow{4}{*}{$\begin{array}{l}\mathrm{S}_{2}(30 \mathrm{~cm} \times 20 \mathrm{~cm}) \\
\left(16.7 \text { plants per } \mathrm{m}^{2}\right)\end{array}$} & $\mathrm{F}_{1}(15 \mathrm{~N}: 20 \mathrm{P}: 20 \mathrm{~K}: 10 \mathrm{~S})$ & 32.77 & 30.98 & 31.88 & 32.41 & 30.64 & 31.52 \\
\hline & $\mathrm{F}_{2}(30 \mathrm{~N}: 40 \mathrm{P}: 40 \mathrm{~K}: 20 \mathrm{~S})$ & 35.29 & 33.38 & 34.34 & 34.91 & 33.01 & 33.96 \\
\hline & $\mathrm{F}_{3}(45 \mathrm{~N}: 60 \mathrm{P}: 60 \mathrm{~K}: 30 \mathrm{~S})$ & 35.91 & 33.96 & 34.94 & 35.51 & 33.59 & 34.55 \\
\hline & Mean & 34.66 & 32.77 & 33.72 & 34.28 & 32.41 & 33.35 \\
\hline \multirow{4}{*}{$\begin{array}{l}\mathrm{S}_{3}(40 \mathrm{~cm} \times 10 \mathrm{~cm}) \\
\left(25 \text { plants per } \mathrm{m}^{2}\right)\end{array}$} & $\mathrm{F}_{1}(15 \mathrm{~N}: 20 \mathrm{P}: 20 \mathrm{~K}: 10 \mathrm{~S})$ & 29.74 & 28.10 & 28.92 & 29.41 & 27.79 & 28.60 \\
\hline & $\mathrm{F}_{2}(30 \mathrm{~N}: 40 \mathrm{P}: 40 \mathrm{~K}: 20 \mathrm{~S})$ & 32.91 & 31.11 & 32.01 & 32.54 & 30.77 & 31.66 \\
\hline & $\mathrm{F}_{3}(45 \mathrm{~N}: 60 \mathrm{P}: 60 \mathrm{~K}: 30 \mathrm{~S})$ & 34.29 & 32.43 & 33.36 & 33.91 & 32.07 & 32.99 \\
\hline & Mean & 32.31 & 30.55 & 31.43 & 31.96 & 30.21 & 31.08 \\
\hline \multicolumn{8}{|c|}{ For Comparing varieties (A) and Nutritional combinations (C) } \\
\hline \multicolumn{2}{|c|}{$\mathrm{F}_{1}(15 \mathrm{~N}: 20 \mathrm{P}: 20 \mathrm{~K}: 10 \mathrm{~S})$} & 30.58 & 28.90 & 29.74 & 30.24 & 28.58 & 29.41 \\
\hline \multicolumn{2}{|c|}{$\mathrm{F}_{2}(30 \mathrm{~N}: 40 \mathrm{P}: 40 \mathrm{~K}: 20 \mathrm{~S})$} & 33.41 & 31.59 & 32.50 & 33.04 & 31.24 & 32.14 \\
\hline \multicolumn{2}{|c|}{$\mathrm{F}_{3}(45 \mathrm{~N}: 60 \mathrm{P}: 60 \mathrm{~K}: 30 \mathrm{~S})$} & 34.29 & 32.43 & 33.36 & 33.91 & 32.07 & 32.99 \\
\hline \multicolumn{2}{|c|}{ Mean } & 32.76 & 30.97 & 31.86 & 32.40 & 30.63 & 31.51 \\
\hline Factor & \multicolumn{2}{|l|}{ SEm士 } & \multicolumn{2}{|c|}{$C D$} & \multicolumn{2}{|c|}{$S E m \pm$} & $C D$ \\
\hline Variety (A) & & \multicolumn{2}{|c|}{1.06} & & 1.00 \\
\hline Ptg. Geom. (B) & \multicolumn{2}{|l|}{0.23} & \multicolumn{2}{|c|}{0.64} & \multicolumn{2}{|c|}{0.21} & 0.62 \\
\hline Nutril. Combn.(C) & \multicolumn{2}{|l|}{0.37} & \multicolumn{2}{|c|}{1.07} & \multicolumn{2}{|c|}{0.35} & 1.01 \\
\hline $\mathbf{A} \times \mathbf{B}$ & \multicolumn{2}{|l|}{ - } & \multicolumn{2}{|c|}{$N S$} & \multicolumn{2}{|c|}{ - } & $N S$ \\
\hline $\mathbf{B} \times \mathbf{C}$ & \multicolumn{2}{|l|}{0.66} & \multicolumn{2}{|c|}{1.91} & \multicolumn{2}{|c|}{0.63} & 1.82 \\
\hline$A \times C$ & 0.70 & & 2.0 & & 0. & & 1.92 \\
\hline$A \times B \times C$ & & & 2.9 & & . & & $N S$ \\
\hline
\end{tabular}

CD: $\mathrm{CD}$ at $5 \%$ level of significance 
Table.4 Seed yield per plant (g) as influenced by variety, planting geometry and nutritional combination during kharif and rabi (pooled data of 2014-15 and 2015-16)

\begin{tabular}{|c|c|c|c|c|c|c|c|}
\hline \multirow{3}{*}{$\begin{array}{l}\text { Planting Geometry } \\
\text { (B) }\end{array}$} & \multirow{3}{*}{$\begin{array}{c}\text { Nutritional Combination } \\
\text { (C) }\end{array}$} & \multicolumn{6}{|c|}{ Variety $(\mathrm{A})$} \\
\hline & & \multicolumn{3}{|c|}{ Kharif } & \multicolumn{3}{|c|}{ Rabi } \\
\hline & & HG 365 & HG 563 & Mean & HG 365 & HG 563 & Mean \\
\hline \multirow{4}{*}{$\begin{array}{l}\mathrm{S}_{1}(30 \mathrm{~cm} \times 10 \mathrm{~cm}) \\
\left(33.3 \text { plants per } \mathrm{m}^{2}\right)\end{array}$} & $\mathrm{F}_{1}(15 \mathrm{~N}: 20 \mathrm{P}: 20 \mathrm{~K}: 10 \mathrm{~S})$ & 15.53 & 13.20 & 14.36 & 13.82 & 11.75 & 12.78 \\
\hline & $\mathrm{F}_{2}(30 \mathrm{~N}: 40 \mathrm{P}: 40 \mathrm{~K}: 20 \mathrm{~S})$ & 17.77 & 15.11 & 16.44 & 15.82 & 13.45 & 14.63 \\
\hline & $\mathrm{F}_{3}(45 \mathrm{~N}: 60 \mathrm{P}: 60 \mathrm{~K}: 30 \mathrm{~S})$ & 19.26 & 16.37 & 17.81 & 17.14 & 14.57 & 15.85 \\
\hline & Mean & 17.52 & 14.89 & 16.21 & 15.59 & 13.25 & 14.42 \\
\hline \multirow{4}{*}{$\begin{array}{l}\mathrm{S}_{2}(30 \mathrm{~cm} \times 20 \mathrm{~cm}) \\
\left(16.7 \text { plants per } \mathrm{m}^{2}\right)\end{array}$} & $\mathrm{F}_{1}(15 \mathrm{~N}: 20 \mathrm{P}: 20 \mathrm{~K}: 10 \mathrm{~S})$ & 18.54 & 15.76 & 17.15 & 16.50 & 14.03 & 15.27 \\
\hline & $\mathrm{F}_{2}(30 \mathrm{~N}: 40 \mathrm{P}: 40 \mathrm{~K}: 20 \mathrm{~S})$ & 21.42 & 18.21 & 19.82 & 19.07 & 16.21 & 17.64 \\
\hline & $\mathrm{F}_{3}(45 \mathrm{~N}: 60 \mathrm{P}: 60 \mathrm{~K}: 30 \mathrm{~S})$ & 22.35 & 18.99 & 20.67 & 19.89 & 16.90 & 18.40 \\
\hline & Mean & 20.77 & 17.66 & 19.21 & 18.49 & 15.71 & 17.10 \\
\hline \multirow{4}{*}{$\begin{array}{c}\mathrm{S}_{3}(40 \mathrm{~cm} \times 10 \mathrm{~cm}) \\
\left(25 \text { plants per } \mathrm{m}^{2}\right)\end{array}$} & $\mathrm{F}_{1}(15 \mathrm{~N}: 20 \mathrm{P}: 20 \mathrm{~K}: 10 \mathrm{~S})$ & 17.81 & 15.14 & 16.47 & 15.85 & 13.47 & 14.66 \\
\hline & $\mathrm{F}_{2}(30 \mathrm{~N}: 40 \mathrm{P}: 40 \mathrm{~K}: 20 \mathrm{~S})$ & 20.35 & 17.30 & 18.83 & 18.11 & 15.40 & 16.76 \\
\hline & $\mathrm{F}_{3}(45 \mathrm{~N}: 60 \mathrm{P}: 60 \mathrm{~K}: 30 \mathrm{~S})$ & 21.63 & 18.38 & 20.00 & 19.25 & 16.36 & 17.80 \\
\hline & Mean & 19.93 & 16.94 & 18.43 & 17.74 & 15.08 & 16.41 \\
\hline \multicolumn{8}{|c|}{ For Comparing varieties (A) and Nutritional combinations (C) } \\
\hline \multicolumn{2}{|c|}{$\mathrm{F}_{1}(15 \mathrm{~N}: 20 \mathrm{P}: 20 \mathrm{~K}: 10 \mathrm{~S})$} & 17.29 & 14.70 & 16.00 & 15.39 & 13.08 & 14.24 \\
\hline \multicolumn{2}{|c|}{$\mathrm{F}_{2}(30 \mathrm{~N}: 40 \mathrm{P}: 40 \mathrm{~K}: 20 \mathrm{~S})$} & 19.85 & 16.87 & 18.36 & 17.67 & 15.02 & 16.34 \\
\hline \multicolumn{2}{|c|}{$\mathrm{F}_{3}(45 \mathrm{~N}: 60 \mathrm{P}: 60 \mathrm{~K}: 30 \mathrm{~S})$} & 21.08 & 17.91 & 19.50 & 18.76 & 15.94 & 17.35 \\
\hline \multicolumn{2}{|c|}{ Mean } & 19.41 & 16.50 & 17.95 & 17.27 & 14.68 & 15.98 \\
\hline Factor & \multicolumn{2}{|l|}{$S E m \pm$} & \multicolumn{2}{|c|}{$C D$} & \multicolumn{2}{|c|}{ SEm+ } & $C D$ \\
\hline Variety (A) & \multicolumn{2}{|l|}{0.41} & \multirow{2}{*}{\multicolumn{2}{|c|}{$\begin{array}{l}1.19 \\
0.90\end{array}$}} & \multicolumn{2}{|c|}{0.37} & 1.06 \\
\hline Ptg. Geom. (B) & \multicolumn{2}{|l|}{0.31} & \multirow{2}{*}{\multicolumn{2}{|c|}{$\begin{array}{l}0.90 \\
1.29\end{array}$}} & \multicolumn{2}{|c|}{0.28} & 0.80 \\
\hline Nutril. Combn.(C) & \multicolumn{2}{|l|}{0.45} & & & \multicolumn{2}{|c|}{0.40} & 1.15 \\
\hline$A \times B$ & \multicolumn{2}{|l|}{0.69} & \multicolumn{2}{|c|}{$\begin{array}{l}1.29 \\
1.99\end{array}$} & \multicolumn{2}{|c|}{ - } & $N S$ \\
\hline B $\times$ C & \multicolumn{2}{|l|}{0.72} & \multicolumn{2}{|c|}{2.09} & \multicolumn{2}{|c|}{0.64} & 1.86 \\
\hline $\mathbf{A} \times \mathbf{C}$ & \multirow{2}{*}{\multicolumn{2}{|c|}{-}} & $N S$ & & r & & $N S$ \\
\hline$A \times B \times C$ & & & $N S$ & & & & 2.87 \\
\hline
\end{tabular}

CD: $\mathrm{CD}$ at $5 \%$ level of significance 
In one of the similar studies, Lakshmi Kalyani (2006) stated that among different cultivars, crude gum content varied significantly and the cultivar RGM 112 produced significantly more gum content and yield hectare ${ }^{-1}$ as compared to HG 563, RGC 1003 and GAUG 9703 in cluster bean. She attributed the same to the genotypic differences.

The interaction effect between planting geometry + nutritional level was significant in both kharif and rabi seasons and in case of both protein as well as gum contents. Both these constituents were superior with the widest spacing + the highest nutritional dose (30 cm x $20 \mathrm{~cm}+45 \mathrm{~N}$ : 60P: 60K: $30 \mathrm{~S} \mathrm{~kg}$ per ha) during kharif and rabi seasons.

The pod yield, seed yield and quality parameters including gum content were found to be maximum during kharif when compared to those during rabi. This indicated that the seed cluster bean crop benefitted much from the warm humid conditions rather than cool dry conditions as prevailed in the respective seasons which might be perhaps in the light of low annual rainfall of the location.

\section{Seed yield per plant (g)}

Significant differences were observed in the seed yield per plant (Table 4) due to variety, planting geometry, nutritional combinations and some of their interactions. Among the varieties HG 365 recorded the highest seed yield per plant both in kharif (19.41 g) and rabi seasons (17.27 g). Planting geometry of $30 \mathrm{~cm} \times 20 \mathrm{~cm}\left(\mathrm{~S}_{2}\right)$ recorded significantly the highest seed yield per plant (kharif $19.21 \mathrm{~g}$; rabi $17.10 \mathrm{~g}$ ) which was on par with $40 \mathrm{~cm} \mathrm{x}$ $10 \mathrm{~cm}\left(\mathrm{~S}_{3}\right)$ (kharif $18.43 \mathrm{~g}$; rabi $16.41 \mathrm{~g}$ ). Application of 45N: 60P: 60K: $30 \mathrm{~S} \mathrm{~kg}$ per ha $\left(\mathrm{F}_{3}\right)$ recorded the highest seed yield per plant (kharif $19.50 \mathrm{~g}$; rabi $17.35 \mathrm{~g}$ ) which was on par with $30 \mathrm{~N}$ : $40 \mathrm{P}: 40 \mathrm{~K}$ : $20 \mathrm{~S} \mathrm{~kg}$ per ha $\left(\mathrm{F}_{2}\right)$ (kharif $18.36 \mathrm{~g}$; rabi $16.34 \mathrm{~g}$ ).
The seed yield on per plant basis was also found to be at highest where there was lowest population density which followed the same trend as mentioned in the above mentioned quality parameters. The plants spaced at the widest spacing in the lowest density might had enough opportunity to absorb sufficient quantities of nutrients in the same time as compared to the other closely spaced plants which might be perhaps a reason for the better performance of such widely spaced plants in terms of bio-chemical and quality parameters.

\section{References}

Baviskar, V. S, Shete, P. G. and Daspute, R. A.2012. Influence of organic fertilizers and sulphur levels on yield, quality and economics of Cluster bean (Cyamopsis tetragonoloba). An Asian Journals of Soil Science. 5 (1): 94-96.

Dwivedi, A. K. and Bapat, P. N. 1998. Sulphur-phosphorus interaction on the synthesis of nitrogenous fraction and oil in soybean. Journal of Indian Society of Soil Science.46 : 254-57.

Ibrahim, M. 2009. Determination of forage production potential of maize sown as a mixture with different legumes under different nitrogen application. Ph.D.Thesis. University of Agriculture. Faisalabad, Pakistan.

Kumawat, B. L, Pathan, A. R. K. and Chahan, R. 2004. Response of taramirra to sulphur and phosphorus application on Typic Pasmmnt. Journal of Indian Society of Soil Science.52: 476-78.

Kumawat, S. M, Dhakar, L. L. and Maliwal, P. L. 2000. Effect of irrigation regimes and nitrogen on yield, oil content and nutrient uptake of soybean (Glycine max L.). Indian Journal of Agronomy. 45: 361- 66.

Lakshmi Kalyani, D. 2006. Performance of rainfed Guar cultivars under varied time of sowing. M.Sc.(Agri.) Thesis. ANGR 
Agricultural University, Tirupati.

Morshed, R. M, Rahman, M. M. and Rahman, M. A. 2008. Effect of nitrogen on seed yield, protein content and nutrient uptake of soybean (Glycine max L.). Journal of Agriculture and Rural Development. 6: 13-17.

Shankaralingappa, B. C, Shivaraj, B. and Viswanatha, K. P. 2000. Interaction effect of phosphorus and sulphur on uptake of nitrogen, phosphorus and potassium and sulphur by cowpea. Journal of Agricultural Science. 13: 295-98.

Sheikh, A. A. 2004. Effect of irrigation intervals, nitrogen and phosphorus application on forage yield, carbohydrates and protein contents of guar in the central region of Saudi
Arabia. Saudi Journal of Biological Science. 11 (1): 3-9.

Singh, R. P, Singh, R. K, Yadhav, P. K, Singh, S.N, Prasad, L. and Singh, J. 2006. Effect of sulphur and molybdenum on yield and quality of blackgram (Vigna mungo L.). Crop Research. 32: 336-38.

Undersander, D. J, Putnam, D. H, Kaminski, A. R, Kelling, K. A, Doll, J. D, Oplinger, E. S. and Gunsolus, J. L. 2006. Alternative Field Crops Manual, University of Wisconsin-Madison. Pp. 34-38.

Yadav, B. K. 2011.Interaction effect of phosphorus and sulphur on yield and quality of clusterbean in typichaplustept. World Journal of Agricultural Sciences. 7 (5): 556-60.

\section{How to cite this article:}

Tagore Naik, M., D. Srihari and Dorajeerao, A.V.D. 2017. Effect of Population Density and Nutrition on Nitrate Reductase Activity in Seed Guar Varieties. Int.J.Curr.Microbiol.App.Sci. 6(12): 3695-3703. doi: https://doi.org/10.20546/ijcmas.2017.612.426 